Bangladesh J. Plant Taxon. 12(2): 19-37, 2005 (December)

\title{
HYDROBIOLOGICAL STUDIES WITHIN THE TEA GARDENS AT SRIMANGAL, BANGLADESH. III. CHLOROPHYCEAE (EXCLUDING DESMIDS)
}

\author{
A. K. M. Nurul Islam $^{*}$ and Haseeb Md. Irfanullah ${ }^{1}$ \\ Department of Botany, University of Dhaka, Dhaka-1000, Bangladesh
}

Key words: Acidic habitats, phytoplankton, periphyton, Chlorophyceae, new records, Bangladesh

\begin{abstract}
A total of 83 algal taxa belonging to 40 genera of Chlorophyceae (excluding desmids) have been recorded from some acidic water bodies within the tea gardens at Srimangal, Maulvi Bazar. Of these 14 are new records for Bangladesh.
\end{abstract}

\section{Introduction}

In the previous two installments of the current series of papers, Islam and Irfanullah have described the aquatic macrophytes (Islam and Irfanullah 2000a) and algal flora (excluding Chlorophyceae) (Islam and Irfanullah 2005) of some selected habitats within the tea gardens of Srimangal, Maulvi Bazar. The present paper deals with algae belonging to the Class Chlorophyceae (excluding desmids) of the same habitats.

\section{Materials and Methods}

For the descriptions of the studied waterbodies and meteorological data of the study area see Islam and Irfanullah (2000a). These habitats were predominantly acidic: Baraoora Lake ( $\mathrm{pH}$ 5.5-6.8), ditch ( $\mathrm{pH}$ 5.8-6.6) and the Burburia River ( $\mathrm{pH}$ 6.0-7.2). A total of 120 algal samples (phytoplankton and periphyton) were collected in winter of 1996 (9 January) and different seasons of 1997 (winter, 6 January; spring, 18 March; rainy season, 20 July and autumn, 20 October). For the sample collection methods, and their preservation and examination see Islam and Irfanullah (2005).

\section{Taxonomic enumeration}

This study reveals a total of 83 chlorophycean taxa belonging to 40 genera. Among these, 14 taxa are newly recorded for Bangladesh (marked by asterisks). Nonetheless, a few algal taxa from this area have already been reported by the same authors for the first time in Bangladesh (Islam and Irfanullah 1998, 2000b), which are not marked in this account.

\footnotetext{
* Corresponding author. ${ }^{1}$ IUCN - The World Conservation Union, Bangladesh Country Office, House \# 11, Road \# 138, Gulshan-1, Dhaka-1212, Bangladesh. E-mail: hmirfanullah@yahoo.co.uk
} 
The results of all the studied taxa are given below with the descriptions and illustrations.

Class: Chlorophyceae; Order: Tetrasporales; Family: Palmellaceae

1. ? Asterococcus limneticus Smith, G.M.

(P1. 1, Fig. 11)

(Smith 1920, 104, 20: 7-10)

Colony d. $40.5 \mu \mathrm{m}$; cell d. 3.4-4.7 $\mu \mathrm{m}$; smaller than the typical. Lake; rainy 1997; few.

2. Asterococcus superbus (Cienk.) Scherffel

(Prescott 1951, 86, 4: 10; Islam 1973, 78, 3: 7-12)

(P1. 1, Fig. 10)

Cell d. with sheath 35-44 $\mu \mathrm{m}$, without sheath $21.6 \mu \mathrm{m}$. Lake; winter 1997; few.

3. Chlamydocapsa ampla (Kütz.) Fott

(P1. 3, Fig. 54)

(Fott 1972, 30, 6: 13; Islam 1973, 78, 3: 5-6 as Gloeocystis ampla Kütz.)

Colony d. 20.2-25.6 $\mu \mathrm{m}$; cell L. $8 \mu \mathrm{m}$, d. 4-5.4 $\mu \mathrm{m}$. Lake; winter 1996 and spring 1997; few.

4. Pseudosphaerocystis lacustris (Lemm.) Novak.

(Islam and Irfanullah 2000b, 116, 1: 6)

Lake; winter 96; few.

\section{Family: Tetrasporaceae}

5. Schizochlamys gelatinosa $\mathrm{A}$. Br.

(Islam 1969, 29, Figs. 35-36; Yamagishi 1998, 65)

Cell d. 12.8-15.5 $\mu$ m. Lake; winter 1997; few.

6. Tetraspora gelatinosa (Vauch.) Desvaux

(Whitford and Schumacher 1973, 15, 3: 15)

(Pl. 1, Figs. 1-4)

Hard, deep green macroscopic colony on rock by the bank of the river subjected to splash; colony d. 1.5-2.0 mm; no pseudocilia, spherical cells in groups embedded in thick mucilage, cell d. 5.4-9.4 $\mu \mathrm{m}$. River; autumn 1997; few.

\section{Family: Coccomyxaceae}

7. Elakatothrix viridis (Snow) Printz : Lake; winter 1997; rare.

Order: Chlorococcales; Family: Chlorococcaceae

8. Desmatractum bipyramidatum (Chod.) Pascher

(Islam and Irfanullah 1998, 92, Figs. 10-15)

Lake: winter 1997 (rare); River: spring 1997 (common).

Family: Dictyosphaeridiaceae

9. Dictyosphaeridium sp. : Lake; winter 1997; rare. 


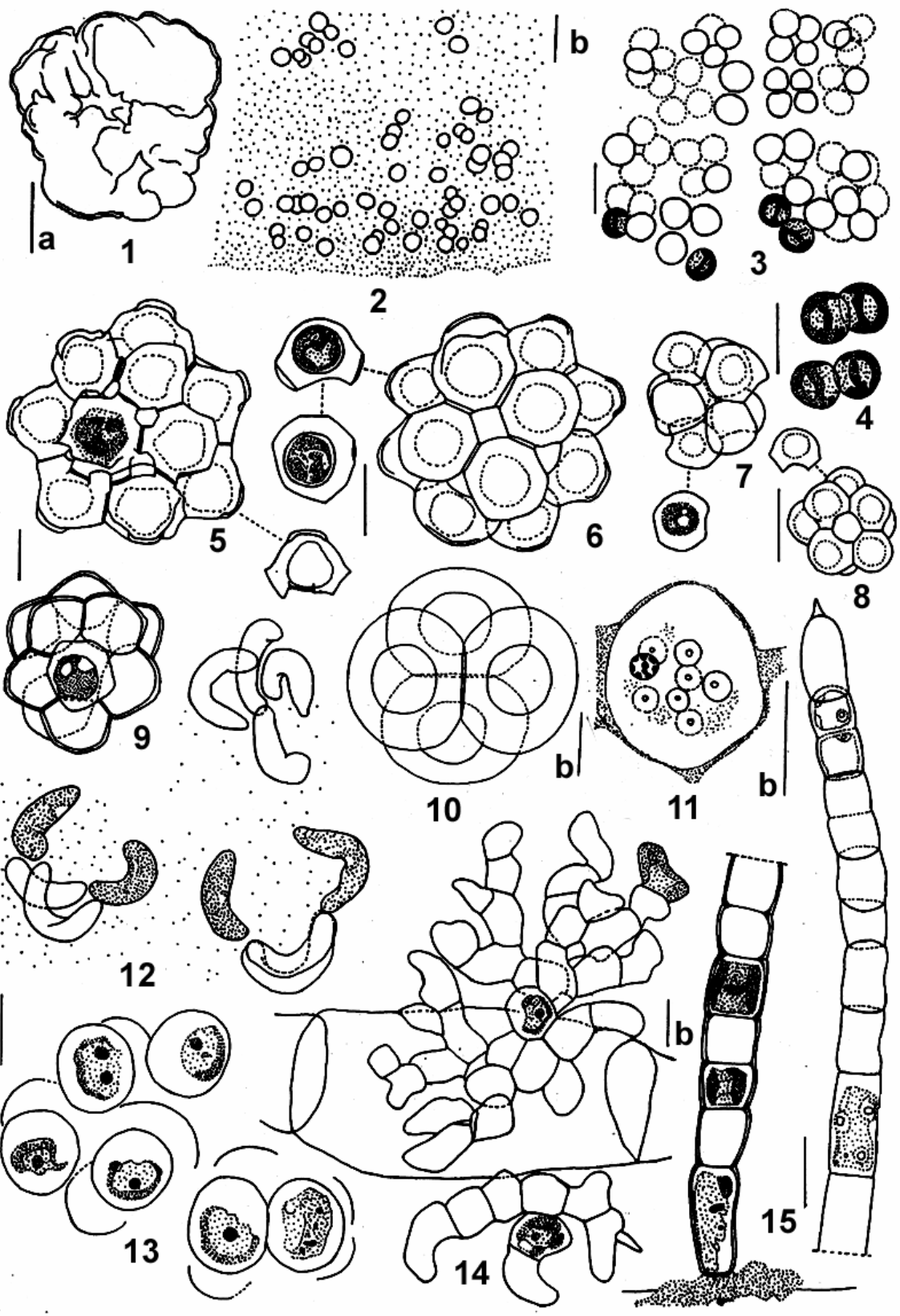

Plate 1 (Figs. 1-15)

Figs. 1-4. Tetraspora gelatinosa (1. a colony, 2. cells on the periphery, 3-4. cells in the middle), 5-6. Coelastrum cambricum, 7. C. sphaericum fa., 8. C. microporum, 9. C. sphaericum, 10. Asterococcus superbus, 11. A. limneticus, 12. Tetrallantos lagerheimii, 13. Schizochlamys gelatinosa, 14. Protoderma viride, 15. Ulothrix tenerrima. (Scales: $\mathrm{a}=0.5 \mathrm{~mm}, \mathrm{~b}=20 \mu \mathrm{m}$, rest $=10 \mu \mathrm{m}$ ) 
10. Dimorphococcus lunatus A. Br.

(P1. 2, Fig. 36)

(Islam 1969, 25, Figs. 24-25)

Cell L. 13.5-23 $\mu \mathrm{m}$, d. 4.7-8 $\mu \mathrm{m}$. Lake; winter 1996; common.

\section{Family: Hydrodictyaceae}

11. Pediastrum duplex Meyen

(P1. 2, Figs. 42-43)

(Islam and Khatun 1966, 99, 6: 141)

Colony d. 40.5-67.5 $\mu \mathrm{m}$; cell L.13.5-18.2 $\mu \mathrm{m}$, d. 13.5-16.2 $\mu \mathrm{m}$. Lake; winter to rainy 1997; rare to few.

12. Pediastrum duplex var. rugulosum Racib.

(Prescott 1951, 224, 49: 3; Islam 1973, 76, 3: 1)

(P1. 2, Fig. 41)

Colony d. $67.5 \mu \mathrm{m}$, cell L. $16.2 \mu \mathrm{m}$, d. 13.5-14.8 $\mu \mathrm{m}$. Lake; spring 1997; few.

13. Pediastrum tetras (Ehr.) Ralfs var. tetraödon (Corda) Hansg. (Pl. 2, Figs. 44-45) (Smith 1920, 174, 48: 13-14, 49: 1-2)

Colony d. 21.3-40 $\mu \mathrm{m}$; cell L. 9.4-12 $\mu \mathrm{m}$, d. 8-13.3 $\mu \mathrm{m}$. Lake; winter 1997; few.

\section{Family: Coelastraceae}

14. Coelastrum cambricum Archer

(Pl. 1, Figs. 5-6) (Smith 1920, 161, 42: 2-3)

16-celled coenobium, d. 33.7-51.3 $\mu \mathrm{m}$; cells are curved from the side view with thickening in the cell wall on the periphery appearing as a flat protrusion, each cell is connected with 3-4-6 other cells, cell d. 10.8-19 $\mu$ m. Lake; winter 1996; common.

15. Coelastrum microporum Näg.

(Pl. 1, Fig. 8)

(Smith 1920, 160, 41: 12-13, 42: 1)

8-celled small coenobium, d. $16.2 \mu \mathrm{m}$; thin cell wall, cell d. $6.7 \mu \mathrm{m}$. Lake; autumn 1997; rare.

16. *Coelastrum sphaericum Näg.

(Pl. 1, Fig. 9)

(Whitford and Schumacher 1973, 43, 11: 19)

10(?)-celled coenobium, d. $32.4 \mu \mathrm{m}$; polygonal cells with uniformly thick cell wall, cell d. $12.2 \mu \mathrm{m}$; parietal chloroplast. Lake; winter 1997; very rare.

17. Coelastrum sphaericum Näg. fa.

(Pl. 1, Fig. 7)

8-celled coenobium, d. 18.2-21.6 $\mu \mathrm{m}$; vegetative cells broadly curved with thin cell wall, d. 7.4-8.7 $\mu \mathrm{m}$. Lake; winter 1996; few.

\section{Family: Oocystaceae}

18. Ankistodesmus falcatus (Corda) Ralfs (Islam and Begum 1970, 244, 3: 85-86; Yamagishi 1998, 69)

(P1. 2, Figs. 39-40)

Cell L. $86.4 \mu \mathrm{m}$, d. $2.7 \mu \mathrm{m}$. Lake; winter 1996, winter and spring 1997; few. 
19. Ankistodesmus spiralis (Turner) Lemm. : Lake; autumn 1997; few.

20. ? Closteriopsis longissima Lemm. var.

(P1. 2, Fig. 37)

Cell L. $144.4 \mu \mathrm{m}$, d. $7.4 \mu \mathrm{m}$, tip d. $2 \mu \mathrm{m}$. Lake; winter 1997; common.

21. Glaucocystis nostochinearum Itz.

(Islam and Irfanullah 1998, 93, Figs. 6-9)

Lake; rainy 1997; common.

22. Kirchneriella sp. : Lake and river; winter 1997; few.

23. Nephrochlamys subsolitaria (G.S. West) Kors.

(Islam and Irfanullah 2000b, 116, 1: 4-5)

Lake; rainy 1997; few. However, Islam and Alfasane (2001) suggested it would be a species of Nephrocytium or Kirchneriella.

24. ? Nephrocytium limneticum Smith, G.M.

(P1. 3, Fig. 60)

(Smith 1950, 261, Fig. 176c)

Slightly curved long cells with blunt poles embedded in thick, heterogeneous mucilage; cells are single or in pairs, cell L. 15.5-17.5 $\mu \mathrm{m}$, d. 6.7-8.2 $\mu \mathrm{m}$. Lake; rainy 1997; rare.

25. Nephrocytium obesum W \& W

(P1. 3, Fig. 61)

(Prescott 1951, 249, 54: 20)

Four slightly curved large cells are loosely enclosed in a thick sheath (d. $2 \mu \mathrm{m})$ without any nodules on it; colony L. $75.6 \mu \mathrm{m}$, d. $58 \mu \mathrm{m}$; cell L. 35-39 $\mu \mathrm{m}$, d. 19-23 $\mu \mathrm{m}$. Lake; winter 1996; rare.

26. Oocystella lacustris (Chod.) Hind.

(P1. 3, Fig. 53)

(Hindak and Moustaka-Gouni 1990, 172, Figs. 7-8; Yamagishi 1998, 73; Smith 1920, 112, 22: 8-9 as Oocystis lacustris)

Colony d. 20.2-21.6 $\mu \mathrm{m}$, cell L. 10-13.5 $\mu \mathrm{m}$, d. 6.7-7.4 $\mu \mathrm{m}$. Lake; rainy 1997; few.

27. Oocystella lacustris (Chod.) Hind. fa.

(P1. 2, Fig. 32)

Colony d. 31.7-34.4 $\mu \mathrm{m}$; cells L. 14.2-22.3 $\mu \mathrm{m}$, d. 11.5-13.5 $\mu \mathrm{m}$; lamellated, hyaline cell wall. Lake; winter 1996; rare.

28. Oocystis borgei Snow

(P1. 2, Fig. 31)

(Smith 1920, 111, 22: 4; Tiffany and Britton 1952, 117, 32: 322)

Colony d. 20.2-21.6 $\mu \mathrm{m}$; cell L. 10.8-12 $\mu \mathrm{m}$, d. 8-10 $\mu \mathrm{m}$. Lake; rainy 1997; common.

29. Oocystis crassa Wittrock : Lake; winter 1997; few. 


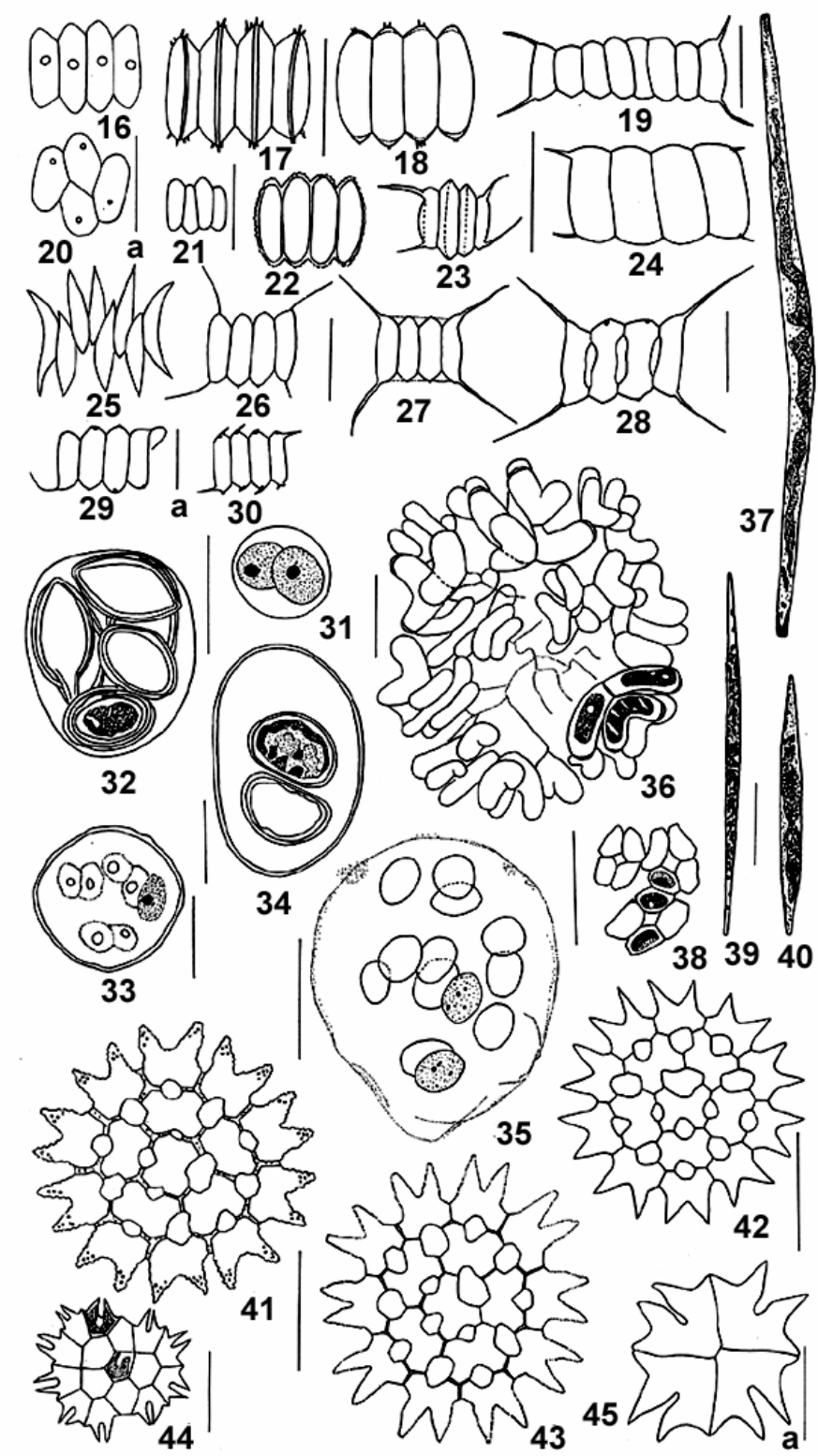

Plate 2 (Figs. 16-45)

Figs. 16, 22. Scenedesmus quadricaudatus var. ecornis, 17. S. brasiliensis, 18. S. denticulatus fa. maximus, 19, 26-27. S. quadricauda, 20. S. arcuatus var. platydiscus, 21. S. bijuga, 23. S. armatus var. spinosus, 24. S. quadricauda var. rectangularis, 25. S. acuminatus, 28. S. perforatus, 29-30. S. longispina var. asymmetricus, 31. Oocystis borgei, 32. Oocystella lacustris fa., 33. Oocystis sp. -1, 34. O. novae-semliae, 35. Oocystis sp. -2, 36. Dimorphococcus lunatus, 37.? Closteriopsis longissima var., 38. Crucigenia crucifera, 39-40. Ankistrodesmus falcatus, 41. Pediastrum duplex var. rugulosum, 42-43. P. duplex, 4445. P. tetras var. tetraödon. (Scales: $\mathrm{a}=10 \mu \mathrm{m}$, rest $=20 \mu \mathrm{m}$ ) 


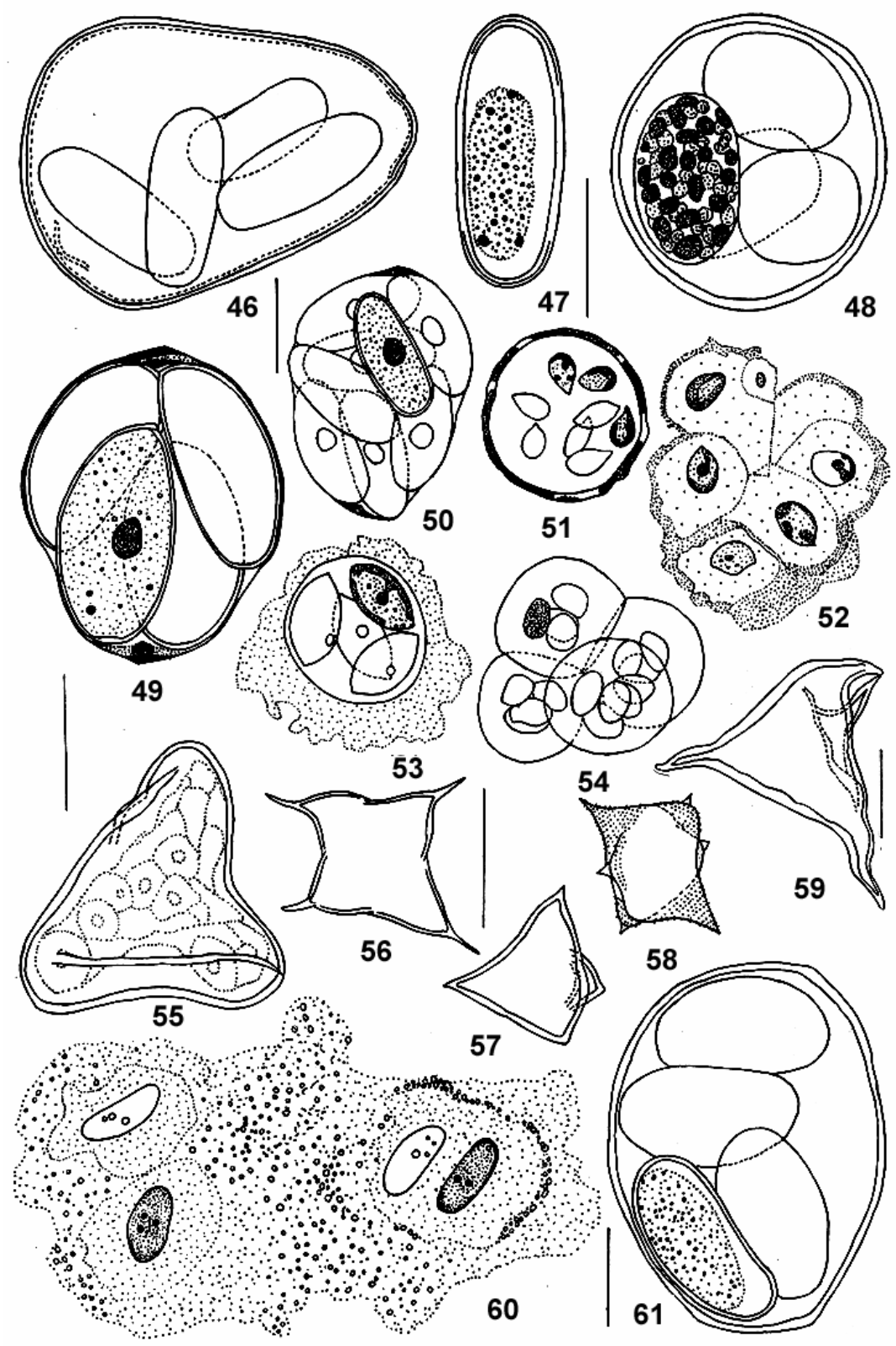

Plate 3 (Figs. 46-61)

Figs. 46-47. Oocystis ? panduriformis var. minor, 48. O. gigas fa., 49. O. solitaria, 50. O. naegelii, 51-52. O. granulata, 53. Oocystella lacustris, 54. Chlamydocapsa ampla, 55. Tetraedron tumidulum, 56. T. regulare var. torsum, 57. T. regulare var. minor, 58. Tetraedron sp., 59. T. regulare, 60. ? Nephrocytium limneticum, 61. $N$. obesum. $($ Scales $=20 \mu \mathrm{m})$ 
30. Oocystis gigas Archer fa.

(P1. 3, Fig. 48) 4-celled globose colony surrounded by a thick, hyaline mucilage sheath, colony d. 46 $\mu \mathrm{m}$; cells broadly elliptic with numerous disc-shaped chloroplasts, cell L. 24.3-27 $\mu \mathrm{m}$, d. $17 \mu \mathrm{m}$. Smaller than Prescott (1951, 244, 51: 13). Lake; winter 1997; rare.

31. *Oocystis granulata Hortobágyi

(Pl. 3, Figs. 51-52)

(Hindak and Moustaka-Gouni 1990, 169, 6: 3)

8-celled spherical colony with thick mucilage sheath $(\mathrm{d} .<1.3 \mu \mathrm{m})$, colony d. 23-24.3 $\mu \mathrm{m}$, each cell may possess individual sheath (d. 13.5-16.2 $\mu \mathrm{m}$ ); elliptical cells with pointed poles, cell L. 5.4-7.4 $\mu \mathrm{m}$, d. 4-4.7 $\mu \mathrm{m}$. Lake; rainy 1997; few.

32. Oocystis naegelii A.Br.

(Tiffany and Britton 1952, 117, 32: 320)

(P1. 3, Fig. 50)

8 -celled colony with thin sheath having two polar nodules, colony L. $48.6 \mu \mathrm{m}$, d. $37.8 \mu \mathrm{m}$; cylindrical cells compact in a colony, cell L. 24.3-25.6 $\mu \mathrm{m}$, d. 10.8-12 $\mu \mathrm{m}$. Lake; rainy 1997; common.

33. Oocystis novae-semliae Wille

(P1. 2, Fig. 34)

(Prescott 1951, 245)

Colony L. 54-55.3 $\mu \mathrm{m}$, d. 36.4-40.5 $\mu \mathrm{m}$; cell L. 19-21.6 $\mu \mathrm{m}$, d. 13.5-14.8 $\mu \mathrm{m}$. Lake; winter 1996, winter and autumn 1997; few.

34. *Oocystis ? panduriformis W \& W var. minor Smith, G.M. (Pl. 3, Figs. 46-47) (Smith 1920, 114, 23: 3)

4-celled pear-shaped colony with thick mucilage sheath having two polar protrusions, colony L. $62 \mu \mathrm{m}$, d. $46 \mu \mathrm{m}$; long elipto-cylindrical cell L. $43 \mu \mathrm{m}$, d. 16.8-18.2 $\mu \mathrm{m}$. Smith (1920) noted the cells to be slightly convex on both lateral sides. Lake; winter 1996; few.

35. *Oocystis solitaria Wittrock

(P1. 3, Fig. 49)

(Tiffany and Britton 1952, 117, 32: 319)

4-celled colony with thin sheath having two polar nodules, colony L. $41.8 \mu \mathrm{m}$, d. 35

$\mu \mathrm{m}$; elliptic cells compact in a colony, cell L. 27-29.7 $\mu \mathrm{m}$, d. 13.5-16.2 $\mu \mathrm{m}$. Lake; rainy 1997 ; common.

36. Oocystis sp. -1

(P1. 2, Fig. 33)

Colony d. 35-48.6 $\mu \mathrm{m}$; cell wall granulated, L. 8-12 $\mu \mathrm{m}$, d. 5.4-8 $\mu \mathrm{m}$. Lake; winter 1997; common.

37. Oocystis sp. -2

(P1. 2, Fig. 35)

Colony L. $48.6 \mu \mathrm{m}$, d. $28.3 \mu \mathrm{m}$; cell L. 6.7-8 $\mu \mathrm{m}$, d. 5.4-6.7 $\mu \mathrm{m}$. Lake; rainy 1997; rare.

38. Selenastrum sp. : River; spring 1997; rare. 
39. Tetraedron regulare Kütz.

(P1. 3, Fig. 59) (Smith 1920, 118, 24: 14)

Cell max. d. with spine 50.7-57.4 $\mu \mathrm{m}$. Lake; winter 1996; rare.

40. *Tetraedron regulare var. minor Reinsch

(P1. 3, Fig. 57) (Skuja 1949, 65, 10: 33)

Cell pyramoidal with curved sides; cell max. d. $25 \mu \mathrm{m}$. Lake; winter 1996; rare.

41. *Tetraedron regulare var. torsum (Turner) Brunnthaler

(Pl. 3, Fig. 56) (Smith 1920, 119, 24: 17-18; Prescott 1951, 269, 61: 8-10)

Quadrangular cell with smooth cell wall, single spine-like process at each angle, cell d. with spine 36.4-41.8 $\mu \mathrm{m}$, without spine 25-30.4 $\mu \mathrm{m}$. Lake; autumn 1997; rare.

42. *Tetraedron tumidulum (Reinsch) Hansg.

(P1. 3, Fig. 55) (Prescott 1951, 270, 61:17-18)

Cell triangular with round angles, sides are concave, cell wall smooth, cell d. 37.8$43.2 \mu \mathrm{m}$. Lake; rainy 1997; rare.

43. Tetraedron sp.

(P1. 3, Fig. 58)

Cell quadrangular from top view, each of the six angles possesses single spin, very minute spine like granules are concentrically arranged on the cell wall around the spine, cell d. with spine 25.6-30.4 $\mu \mathrm{m}$. Paddy field; autumn 1997; rare.

\section{Family: Scenedesmaceae}

44. Crucigenia crucifera (Wolle) Collins

(Smith 1920, 145, 36: 6; Whitford and Schumacher 1973, 54, 14: 37)

(P1. 2, Fig. 38)

Colony L. 12-14.2 $\mu \mathrm{m}$, d. 8.8-10.8 $\mu \mathrm{m}$; cell L. 5.4-8 $\mu \mathrm{m}$, d. 3.4-4.7 $\mu \mathrm{m}$. Lake; autumn 1997; rare.

45. Scenedesmus acuminatus (Lager.) Chodat

(P1. 2, Fig. 25)

(Islam and Khatun 1966, 99, Fig. 79)

Colony L. $21.6 \mu \mathrm{m}$; cell L. 14.8-20.2 $\mu \mathrm{m}$, d. 2.7-4 $\mu \mathrm{m}$. Lake; spring 1997; few.

46. Scenedesmus arcuatus var. platydiscus Smith, G.M.

(P1. 2, Fig. 20)

(Islam and Begum 1970, 251, 5: 140-141)

Colony L. $11 \mu \mathrm{m}$; cell L. 7.4-8 $\mu \mathrm{m}$, d. $5.4 \mu \mathrm{m}$. Lake; winter 1997; few.

47. Scenedesmus armatus var. spinosus Fritsch \& Rich

(Pl. 2, Fig. 23)

(Hegewald and Silva 1988, 102, Fig. 161)

Colony L. $13.5 \mu \mathrm{m}$; cell. L. 9.4-14.8 $\mu \mathrm{m}$, d. $3.4 \mu \mathrm{m}$. Lake; winter 1996; rare.

48. Scenedesmus bijuga (Turp.) Lagerheim

(P1. 2, Fig. 21)

(Islam and Begum 1970, 252, 5: 147, 149-150)

Colony L. $13.5 \mu \mathrm{m}$; cell L. 10.8-12 $\mu \mathrm{m}$, d. $4 \mu \mathrm{m}$. Lake; winter 1997; rare. 
49. Scenedesmus brasiliensis Bohlin

(Hegewald and Silva 1988, 131, Fig. 214)

(P1. 2, Fig. 17)

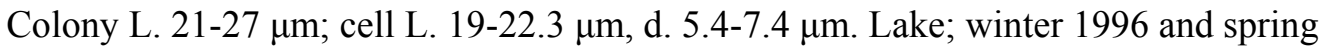
1997; few.

50. *Scenedesmus denticulatus fa. maximus Uherk

(Pl. 2, Fig. 18)

(Hagewald and Silva 1988, 208, Fig. 330)

Colony L. $25.6 \mu \mathrm{m}$; cell L. 20.2-21.6 $\mu \mathrm{m}$, d. $6.7 \mu \mathrm{m}$. Lake; winter 1996; rare.

51. Scenedesmus longispina Chod. var. asymmetricus Hortob.

(Hortobágyi 1960, 181, 27-28: 315-324)

Colony L. 13.5-16.8 $\mu \mathrm{m}$; cell L. 10.2-12.8 $\mu \mathrm{m}$, d. 3.4-6 $\mu \mathrm{m}$; terminal cells have thin, curved spines at alternate tips, spine L. $5.4 \mu \mathrm{m}$. Lake; spring 1997; few.

52. Scenedesmus perforatus Lemm.

(P1. 2, Fig. 28)

(Hegewald and Silva 1988, 388, Fig. 625)

Colony L. $28.3 \mu \mathrm{m}$; cell L. 19-19.7, d. 6.7-8 $\mu \mathrm{m}$. Close to var. pologranulatus Teil., although not separable from the typical (Hegewald and Silva 1988). Lake; spring 1997; few.

53. Scenedesmus quadricauda (Turp.) Bréb.

(Pl. 2, Figs. 19, 26-27)

(Hegewald and Silva 1988, 428, Fig. 687)

Colony L. 21-47.2 $\mu \mathrm{m}$; cell L. 16.2-18 $\mu \mathrm{m}$, d. 5-6.7 $\mu \mathrm{m}$. Lake; autumn and winter 1997; few.

54. *Scenedesmus quadricauda var. rectangularis G.S. West

(P1. 2, Fig. 24)

(Hegewald and Silva 1988, 462, Fig. 746)

Colony L. $33.7 \mu \mathrm{m}$; cell L. $17.5 \mu \mathrm{m}$, d. 8-9.4 $\mu \mathrm{m}$. It resembles S. quadricauda var. quadrispina (Chodat) Smith, G.M. Lake; autumn 1997; rare.

55. *Scenedesmus quadricaudatus var. ecornis Ehr. ex Ralfs $\quad$ (Pl. 2, Figs. 16, 22) (Hegewald and Silva 1988, 468, Fig. 756)

4-celled colony, L. $20.2 \mu \mathrm{m}$; cell L. 13.5-17 $\mu \mathrm{m}$, d. 4.7-5.4 $\mu \mathrm{m}$, cell wall smooth or granulated. Lake; rainy and autumn 1997; rare.

56. Tetrallantos lagerheimii Telling

(Pl. 1, Fig. 12)

(Islam 1969, 27, Figs. 31-33)

Cell L. 12-14.8 $\mu \mathrm{m}$, d. $4 \mu \mathrm{m}$. Lake; winter 97; rare.

\section{Order: Ulotrichales; Family: Ulotrichaceae}

57. Schizomeris leibleinii Kütz.: Lake; on snail, among Cladophora; rainy 1997; few.

58. Ulothrix tenerrima Kütz.

(P1. 1, Fig. 15)

(Islam and Zaman 1974, 87, 1: 3, 18-19) 
Cell L. 5.4-17.5 $\mu \mathrm{m}$, d. 6.7-8 $\mu \mathrm{m}$. Lake; on snail, among Cladophora spp.; rainy 1997; few.

\section{Family: Cylindrocapsaceae}

59. Cylindrocapsa geminella Wolle (Islam and Irfanullah, 2000b, 116, 1: 9-11)

Lake; primarily epiphytic but free-floating after maturation; winter and rainy 1997; few to common.

\section{Order: Chaetophorales; Family: Chaetophoraceae}

60. Chaetophora elegans (Roth) C.A. Agardh

(P1. 4, Fig. 65) (Islam and Ahia 1964, 105, Fig. 14)

Colony d. 2.5-3 mm; cell dimension: main axis 24.3-48.6 $\times$ d. 13.5-6.7 $\mu \mathrm{m}$, branch 10.8-27 $\times$ 4.7-7.4 $\mu \mathrm{m}$; seta d. 2-2.7 $\mu \mathrm{m}$. River; colonies free-floating or epilithic; winter and autumn 1997; common.

61. Protoderma viride Kütz.

(P1. 1, Fig. 14) (Printz 1964, 290, 90: 1-2)

Cell d. 13.5-19 $\mu \mathrm{m}$, L. 1-2 times than breadth. Lake; on Oedogonium sp.; winter 1997; few to common.

62. Pseudopleurococcus printzii Vischer

(P1. 4, Fig. 69) (Printz 1964, 278, 86: 1-9)

Cell dimension: apical 19-27 × 16.2-20.2 $\mu \mathrm{m}$, intercalary 16.2-27 $\times 21.6 \mu \mathrm{m}$. Lake; winter 1996 and rainy 1997; rare to few.

63. *Pseudulvella americana (Snow) Wille

(Bourrelly 1972, 53: 4-8)

(P1. 6, Fig. 80)

Thallus microscopic, epiphytic on Chara fibrosa, enclosed in gelatinous envelop, irregular margin, thallus size $173 \times 109 \mu \mathrm{m}$; cell are irregular in shape, roundish polygonal to true polygonal, cell L. 7.4-14.8 $\mu \mathrm{m}$, d. 6.7-10 $\mu \mathrm{m}$; chloroplast parietal. Lake; winter 1997; few.

64. Stigeoclonium aestivale (Hazen) Collins

(P1. 4, Figs. 62-63)

(Printz 1964, 144, 34: 1)

Cell dimension: main axis 18-59 $\times 5.4-7.4 \mu \mathrm{m}$, branch $13.5-27 \times 5.4 \mu \mathrm{m}$, basal 5.4$10.8 \times 4-8 \mu \mathrm{m}$, seta d. 3.4-4 $\mu \mathrm{m}$. Lake and ditch; on Oedogonium sp.; autumn to spring 1997; rare to common.

65. Stigeoclonium curvirostrum Skuja

(P1. 4, Fig. 64)

(Skuja 1949, 73, 12: 4-8)

Cell dimension: primary branch $13.5-40.5 \times 4.7-7.4 \mu \mathrm{m}$, secondary branch 14.8-32.4 $\times$ 4.7-7.4 $\mu \mathrm{m}$, basal 8-16.2 $\times 8$-10.8 $\mu \mathrm{m}$. Lake; epiphytic; winter, few. 

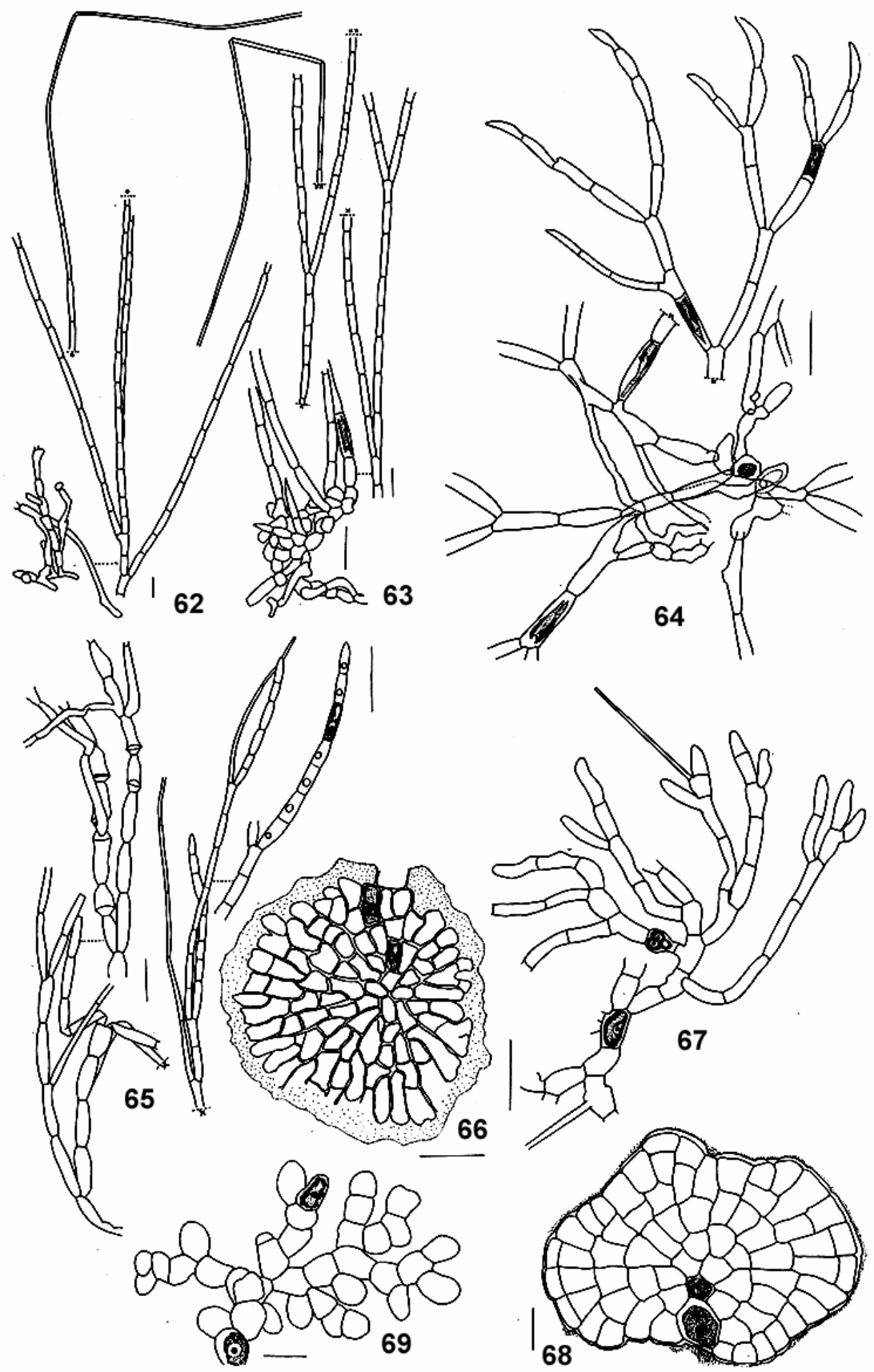

Plate 4 (Figs. 62-69)

Figs. 62-63. Stigeoclonium aestivale, 64. S. curvirostrum, 65. Chaetophora elegans, 66. C. soluta var. soluta, 67. C. conchata, 68. C. scutata, 69. Pseudopleurococcus printzii. (Scales: Figs. $62-63=20 \mu \mathrm{m}$, Fig. $67=$ $50 \mu \mathrm{m}$, rest $=30 \mu \mathrm{m})$ 


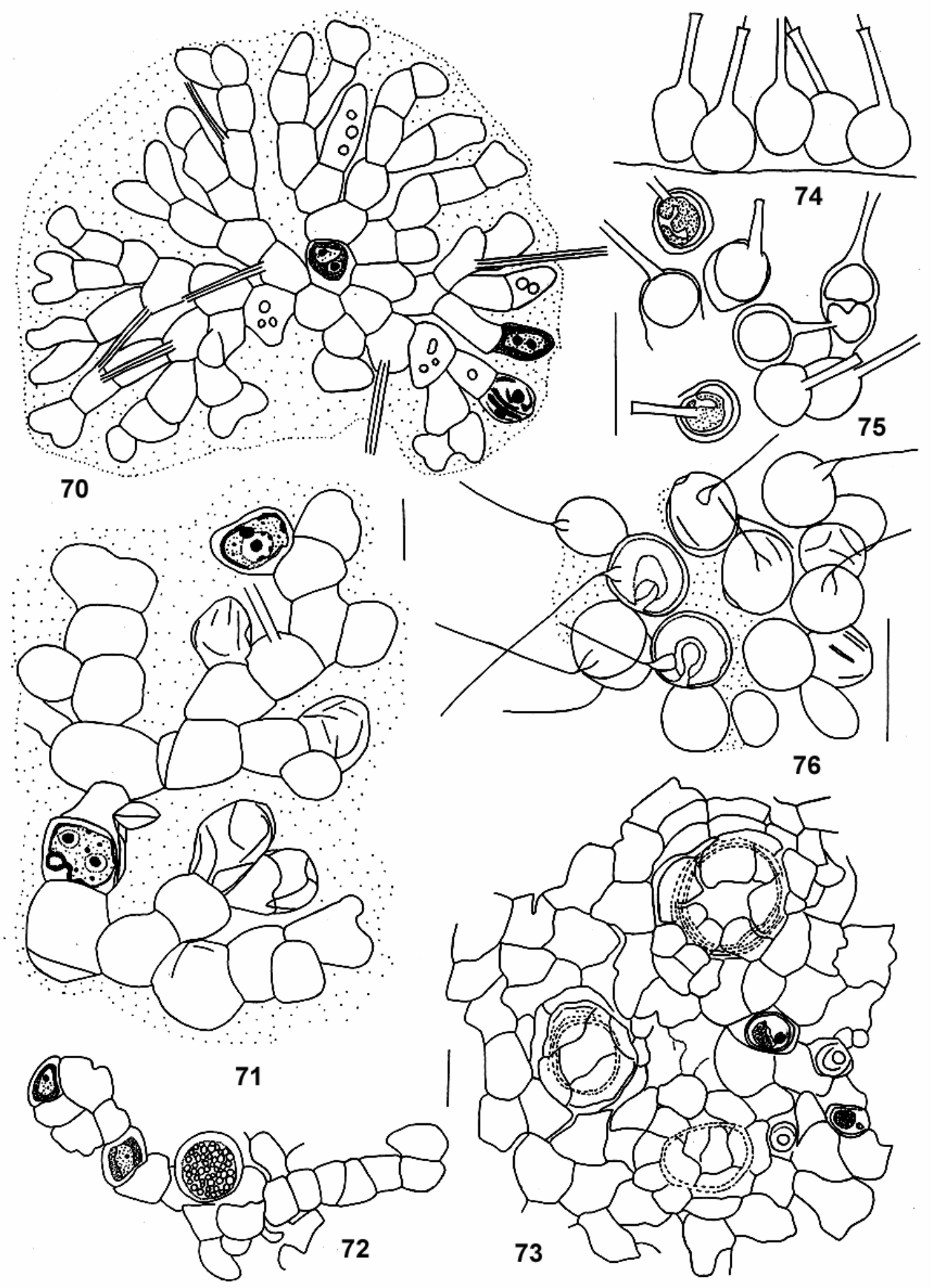

Plate 5 (Figs. 70-76)

Figs. 70-71. Coleochaete conchata, 72-73. C. nitellarum, 74. Chaetosphaeridium globosum, 75. C. pringsheimii, 76. C. ? pringsheimii. (Scales $=20 \mu \mathrm{m})$. 
66. Stigeoclonium geraldii Islam: Lake; on Nymphoides indicum petiole; rainy 1997; rare.

67. *Ulvella frequens Butcher

(Bourrelly 1972, 288, 53: 9)

(P1. 6, Fig. 79)

Thallus microscopic, epiphytic on stems of Panicum paludosum, forms one-cell-thick pseudoparenchymatous disc, irregular in outline, filaments radiating near periphery; cells irregularly angular in shape, central cell L. 13.5-21 $\mu \mathrm{m}, \mathrm{d}$. 9.4-16.2 $\mu \mathrm{m}$, peripheral cell L. 8-19 $\mu \mathrm{m}$, d. 8-13.5 $\mu \mathrm{m}$; tip cells triangular; chloroplast parietal. Lake; winter 1997; few.

\section{Order: Coleochaetales; Family: Aphanochaetaceae}

68. Aphanochaete repens $\mathrm{A}$. $\mathrm{Br}$.

(P1. 6, Figs. 77-78) (Islam 1974, 35, 3: 15-16)

Thallus L. $>200 \mu \mathrm{m}$; cell dimensions: apical 10.8-13.5 $\times 5.4-6 \mu \mathrm{m}$, intercalary 12$15.5 \times 6-8.8 \mu \mathrm{m}$; seta base d. 2.7-4 $\mu \mathrm{m}$. Lake; on Oedogonium sp.; autumn to spring 1997; rare to common.

\section{Family: Coleochaetaceae}

69. Coleochaete conchata Moeb.

(P1. 4, Fig. 67, Pl. 5, Figs. 70-71)

(Khan and Islam 2000, 15, 1: 1, 2: 2-4, 4: 11)

Cell L. 27-32.4 (-40.5) $\mu \mathrm{m}$, d. (6.7-) 10.8-16.2 $\mu \mathrm{m}$; seta L. 32.4-54 $\mu \mathrm{m}$. Lake; on Nymphoides indicum petiole; rainy and autumn 1997; few to common.

70. Coleochaete nitellarum Jost

(P1. 5, Figs. 72-73)

(Islam 1974, 36, 1: 4-7, 2: 8-12)

Lake; on Chara fibrosa; winter 1996 and 1997; few.

71. Coleochaete scutata Bréb.

(P1. 4, Fig. 68)

(Islam 1974, 37, 1: 1-3)

Thallus d. 153-218 $\mu \mathrm{m}$; cell L. 14.8-32.4 $\mu \mathrm{m}$, d. 13.5-27 $\mu \mathrm{m}$. Lake; epiphytic; winter and rainy 1997; few to common.

72. Coleochaete soluta (Bréb.) Pringsh. var. soluta

(P1. 4, Fig. 66) (Islam 1974, 37, 3: 19-20)

Thallus d. 113-122 $\mu \mathrm{m}$; cell L. 10.8-27, d. 8-12 $\mu \mathrm{m}$. Lake; on Nymphoides indicum petiole; rainy and autumn 1997; few.

\section{Family: Chaetosphaeridaceae}

73. Chaetosphaeridium globosum (Nordst.) Klebahn

(P1. 5, Fig. 74)

(Islam 1974, 38, 1: 7a)

Cell L. 10.8-12.8 $\mu \mathrm{m}$, d. 10-12 $\mu \mathrm{m}$; seta L. $13.5 \mu \mathrm{m}$. Lake; winter 1996, winter to rainy 1997; rare to few. 


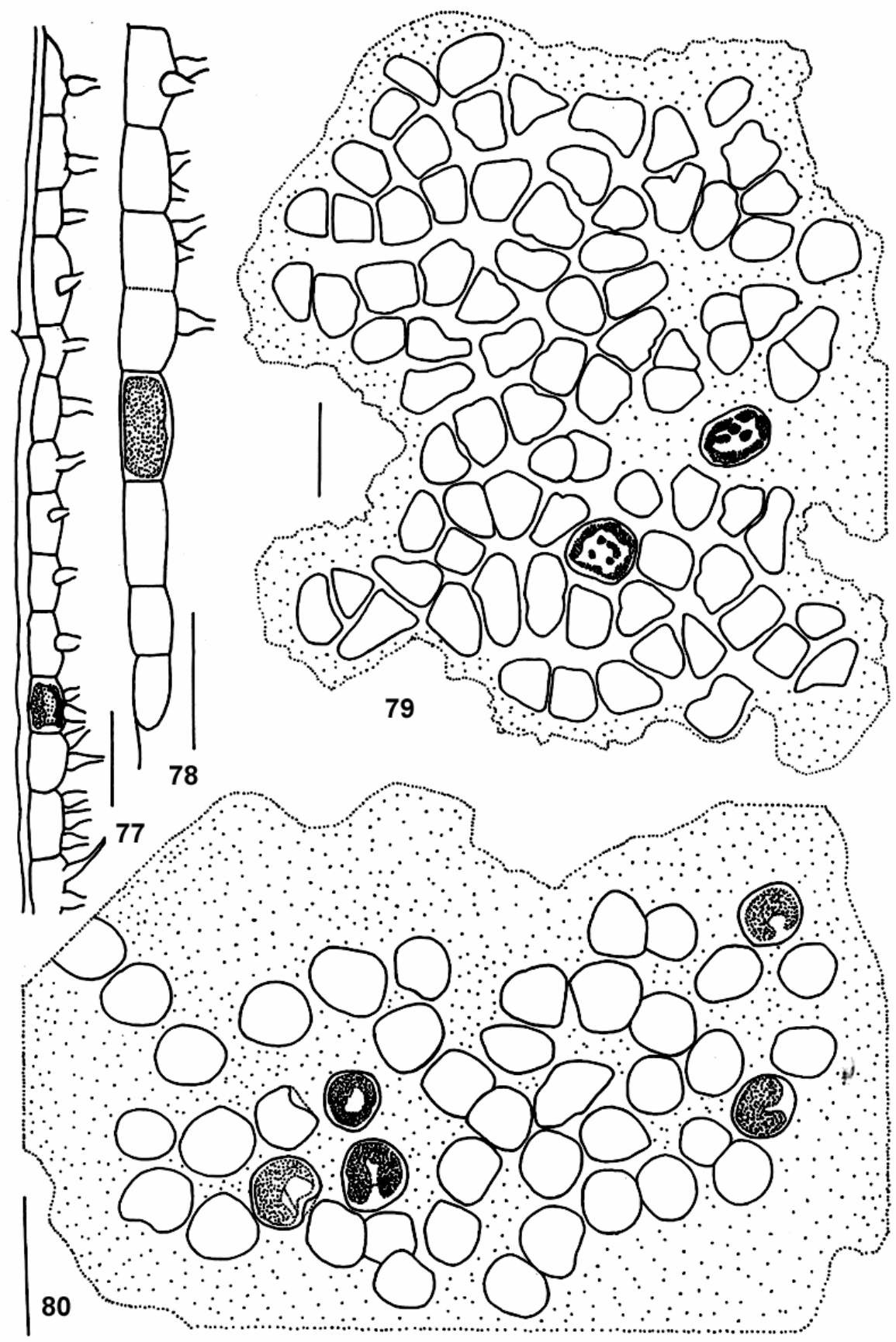

Plate 6 (Figs. 77-80)

Figs. 77-78. Aphanochaete repens, 79. Ulvella frequens, 80. Pseudulvella americana. $($ Scales $=20 \mu \mathrm{m})$ 
74. Chaetosphaeridium pringsheimii Klebahn

(P1. 5, Fig. 75)

(Printz 1964, 332, 104: 1-2; Bourrelly 1972, 323, 63: 10, 64: 1)

Cell d. 8.8-10.8 $\mu \mathrm{m}$, seta L. max. $12.5 \mu \mathrm{m}$, d. $1.3 \mu \mathrm{m}$; utricle present. Epithytic on Oedogonium sp. along with Stigeoclonium aestivale. Lake; winter 1997; rare.

75. Chaetosphaeridium ? pringsheimii Klebahn

(Pl. 5, Fig. 76)

(Tiffany and Britton 1952, 44, 6: 59)

Globose cells irregularly arranged on the substrate, d. 8-13.5 $\mu \mathrm{m}$; seta base d. 2-2.7 $\mu \mathrm{m}$; utricle not clearly visible. Lake; on Nymphoides indicum petiole; rainy 1997; rare.

\section{Order: Oedogoniales; Family: Oedogoniaceae}

76. Bulbochaete spp. : Lake; year round; common; lacked mature oospores.

77. Oedogonium spp. : Lake and ditch; year round; common; lacked mature oospores.

\section{Order: Cladophorales; Family: Cladophoraceae}

78. Cladophora profunda Brand

(P1. 7, Fig. 82) (Islam and Zaman 1975, 48, 2: 18)

Cell dimensions: main axis, 170-740 $\times 32-43 \mu \mathrm{m}$ and branch, $105-570 \times 22-39 \mu \mathrm{m}$; sporangia dimension $128-269 \times 32-45 \mu \mathrm{m}$. Lake; on snail; rainy 1997 ; few.

79. *Cladophora sterrocladia Skuja

(P1. 7, Fig. 81) (Skuja 1949, 94, 37: 1-7)

Red-brown branched thallus growing on snail, primary branches are opposite but secondary branches are unilateral, few successive unilateral branches can also be seen on the main axis; cell dimension: main axis $134-205 \times 35-51 \mu \mathrm{m}$, primary branch 147-307 $\times 26-45 \mu \mathrm{m}$; reproduction was not observed. Lake; rainy 1997; few.

\section{Order: Zygnemales; Family: Zygnemaceae}

80. *Mougeotia viridis (Kütz) Wittrock fa.

(P1. 7, Fig. 83) Zygospores formed in the conjugation tube, quadratic, wall smooth, sides concave, corners retuse, dimension $35 \times 28 \mu \mathrm{m}$. Differs from Prescott (1951, 306, 71: 8-10). Lake; winter 1997; common.

81. Mougeotia sp.

(Pl. 7, Figs. 84-85) Azygospores formed in the sporangia, cubico-cylindrical, dimension $24 \times 19 \mu \mathrm{m}$. Lake; winter and autumn 1997; few to common.

82. Spirogyra spp. : Lake and river; year round; common; lacking mature zygospores.

83. Zygnema sp. : River; spring 1997; few. 


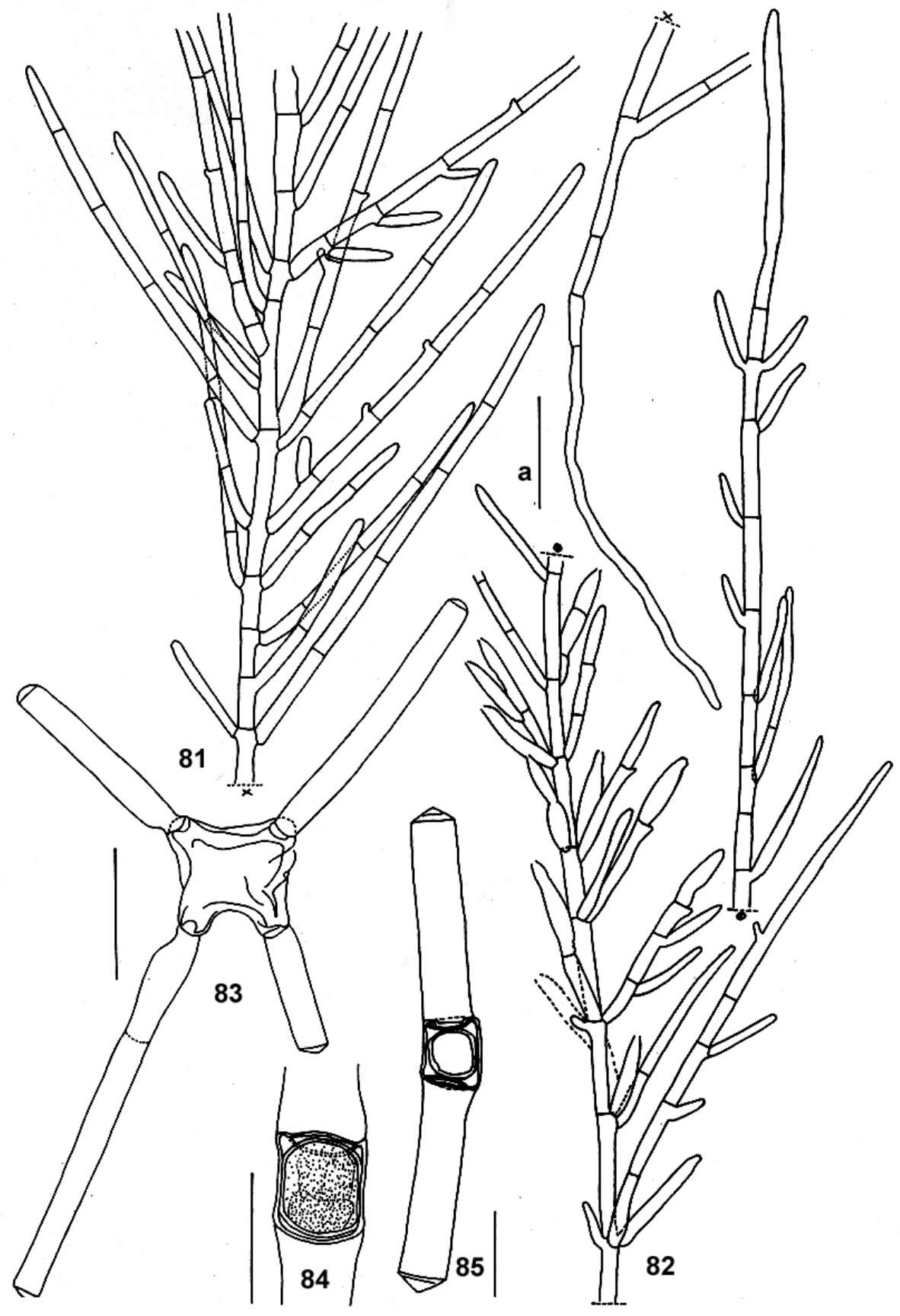

Plate 7 (Figs. 81-85)

Fig. 81. Cladophora sterrocladia, 82. C. profunda, 83. Mougeotia viridis fa., 84-85. Mougeotia sp. (Scales: a $=300 \mu \mathrm{m}$, rest $=30 \mu \mathrm{m})$ 


\section{Acknowledgements}

We are grateful to A.F.M. Badrul Alam, the then Director of Bangladesh Tea Research Institute (BTRI), Srimangal, for providing the logistic and laboratory support during this study, and also to his colleagues who extended their help in the laboratory and in supplying necessary information. Thanks also due to the authority of the James Finley $\&$ Co. for granting permission in collecting samples from the aquatic habitats within its gardens.

\section{References}

Bourrelly, P. 1972. Les algues d'eau douce. Initiation a la systématique I: Les Algues Vertes. Editions N. Boubée \& Co. Paris, pp. 572.

Fott, V.B. 1972. Das Phytoplankton des Süsswassers : Systematik und Biologie. 6 Teil. Chlorophyceae; Ordnung : Tetrasporales. E. Schw. Verlags. Stuttgart, pp. 116 + pls. 1-47.

Hegewald, E. and Silva, P.C. 1988. Annotated catalogue of Scenedesmus and nomenclaturally related genera, including original descriptions and figures. Bibl. Phyc. 80: 1-587.

Hindak, F. and Moustaka-Gouni 1990. Planktic Coccal and Tichal Green algae of Lake Volvi, Greece. Folia Geobot. Phyto-Taxon. PRAHA 25(2): 159-196.

Hortobágyi, von T. 1960. Algen aus den Fischteichen von Buzsák III.: Scenedesmus - Arten. Nova Hedwigia 2(1+2): 173-190 + pls. 22-34.

Islam, A.K.M. Nurul 1969. Some rare planktonic green algae found in East Pakistan. Pakistan J. Bot. 1(1+2): 19-32.

Islam, A.K.M. Nurul 1973. Freshwater algae of Bangladesh. I. Chlorophyceae, Xanthophyceae and Chrysophyceae. Dacca Univ. Stud. B 21(1): 69-84.

Islam, A.K.M. Nurul 1974. Freshwater algae of Bangladesh. IV. Aphanochaete, Coleochaete and Chaetosphaeridium. Bangladesh J. Bot. 3(1): 35-43.

Islam, A.K.M. Nurul and Ahia, A.N.M. 1964. Contribution to the knowledge of Chaetophoraceae of Dacca district. Pak. J. Biol. \& Ag. Sc. 7(1): 103-110.

Islam, A.K.M. Nurul and Alfasane, M.A. 2001. New records of some green planktonic algae for Bangladesh: Phcotus, Planktosphaeria and Nephrochlamys. Bangladesh J. Plant Taxon. 8(2): 51-56.

Islam, A.K.M. Nurul and Begum, Z.T. 1970. Studies on the phytoplankton of Dacca District. J. Asiatic Soc. Pak. 15(3): 227-271 + 8 pls.

Islam, A.K.M. Nurul and Irfanullah, H.M. 1998. New records of three green algal genera for Bangladesh: Desmatractum, Glaucocystis and Groenbladia. Bangladesh J. Plant Taxon. 5(1): 91-95.

Islam, A.K.M. Nurul and Irfanullah, H.M. 2000a. Hydrobiological studies within the tea gardens at Srimangal, Bangladesh. I. Aquatic macrophytes. Bangladesh J. Plant Taxon. 7(1): 29-42.

Islam, A.K.M. Nurul and Irfanullah, H.M. 2000b. New records of eleven algal taxa for Bangladesh. Bangladesh J. Bot. 29(2): 115-120.

Islam, A.K.M. Nurul and Irfanullah, H.M. 2005. Hydrobiological studies within the tea gardens at Srimangal, Bangladesh. II. Algal flora (excluding Chlorophyceae). Bangladesh J. Plant Taxon. 12(1): 33-52.

Islam, A.K.M. Nurul and Khatun, M. 1966. Preliminary studies on the phytoplankton of polluted waters. Sci. Res. 3(2): 95-109. 
Islam, A.K.M. Nurul and Zaman, A.M.S. 1974. Freshwater algae of Bangladesh. VIII. Ulotrichales. Dacca Univ. Stud. B. 22(2): 83-98.

Islam, A.K.M. Nurul and Zaman, K.M. 1975. Limnological studies of the River Buriganga III. Biological aspect. J. Asiatic Soc. Bangladesh (Sc.) 1(1): 45-65.

Khan, M.R. and Islam, A.K.M. Nurul 2000. New records of four Coleochaete species (Chlorophyta) for Bangladesh. Bangladesh J. Plant Taxon. 7(1): 15-27.

Prescott, G.W. 1951. Algae of the Western Great Lakes Area. Cranbrook Inst. Sci. Bull. No. 31, pp. 846.

Printz, H. 1964. Die Chaetophoralen der Binnengewasser. Hydrobiologia 24 (1/3): 1-376.

Skuja, von H. 1949. Zur Süsswasseralgen-flora Burmas. Nova Acta Reg. Soc. Sci. Upsaliensis Ser. IV. 14(5): $1-188+39$ pls.

Smith, G.M. 1920. Phytoplankton of the inland lakes of Wisconsin. Part I. Myxophyceae, Phaeophyceae, Heterokonteae, and Chlorophyceae exclusive of the Desmidiaceae. Wisconsin Geological and Natural History Survey, Bulletin No. 57, Sci. Ser. No. 12, 1-243 pp.

Smith, G.M. 1950. The Freshwater Algae of the United States. McGraw-Hill Book Co. Inc. N.Y. 719 pp.

Tiffany, L.H. and Britton, M.E. 1952. The Algae of Illinois. The University of Chicago Press, Chicago, pp. 407.

Whitford, L.A. and Schumacher, G.J. 1973. A Manual of Fresh-water Algae. Sparks Press, Raleigh N.C., pp. 324.

Yamagishi, T. 1998. Guide Book to Photomicrographs of the Freshwater Algae. Uchida, Rokakuho, Japan, pp. 132. 